\title{
Desempenho em leitura, ordenação temporal, motivação e queixas escolares: estudo preliminar
}

\author{
Reading performance, temporal ordering, motivation and school \\ complaints: preliminary study
}

\author{
Cintia Alves de Souza' (D), Andrezza Gonzalez Escarce² (i), Stela Maris Aguiar Lemos ${ }^{3}$ (1)
}

\section{RESUMO}

Objetivo: verificar a associação entre o desempenho em leitura de crianças e adolescentes e a presença de queixas escolares, habilidade auditiva de ordenação temporal e motivação escolar. Métodos: estudo observacional analítico transversal, com amostra de conveniência composta por 36 participantes. Os instrumentos de coleta foram questionário de caracterização, Critério de Classificação Econômica do Brasil, avaliação auditiva, testes de Memória para Sons Verbais, Não Verbais em Sequência, Padrão de Frequência e de Duração, Desempenho Escolar, as Provas de Avaliação dos Processos de Leitura e a Escala para Avaliação da Motivação Escolar Infantojuvenil. A variável resposta deste estudo foi "processos de leitura". Os testes Quiquadrado de Pearson e Exato de Fisher foram utilizados para as análises de associação. Resultados: as análises de associação revelaram que a maioria dos participantes com alteração nas tarefas de leitura apresentou, também, adequação da ordenação temporal simples e inadequação da ordenação temporal complexa. Não houve associação com significância estatística entre o desempenho em leitura e a presença de queixas escolares, habilidade auditiva de ordenação temporal e motivação escolar. Contudo, observou-se que a maioria dos participantes com alteração na leitura apresentou queixa de dificuldades escolares, baixo desempenho acadêmico e motivação escolar média. Conclusão: embora não tenha indicado significância estatística na maioria das associações realizadas, o presente estudo evidenciou que há relação entre o desempenho em leitura de crianças e adolescentes e a presença de queixas escolares, habilidade auditiva de ordenação temporal e motivação escolar.

Palavras-chave: Percepção auditiva; Testes auditivos; Leitura; Criança; Motivação

\begin{abstract}
Purpose: To verify the association between reading performance of children and adolescents and the presence of school complaints, hearing ordering ability and school motivation. Methods: Cross-sectional observational study with a convenience sample composed of 36 participants. The instruments used were the Sociodemographic and School Characterization Questionnaire, the Economic Classification Criterion of Brazil, the auditory evaluation, the Memory Tests for Verbal and Non-verbal Sounds in Sequence, the Frequency and Duration Pattern Tests, the School Performance Test, the Assessment of Reading Processes and the Scale for Assessing Children's School Motivation. The response variable in this study was the reading process. Pearson's Chi-square and Fisher's Exact tests were used for association analyzes. Results: The association analysis revealed that most participants who presented changes in reading tasks also presented adequacy of simple temporal ordering and inadequacy in complex temporal ordering. There was no statistically significant association between reading performance and the presence of school complaints, temporal ordering auditory ability and school motivation. However, it was observed that most participants with reading disorders complained of school difficulties, poor academic performance, average school motivation, adequacy of simple temporal ordering and inadequacy of complex temporal ordering. Conclusion: Although it did not show statistical significance in most of the associations performed, the present study showed that there is an relationship between the reading performance of children and adolescents and the presence of school complaints, temporal ordering auditory ability and school motivation.
\end{abstract}

Keywords: Auditory perception; Hearing tests; Reading; Child; Motivation

Trabalho realizado na Universidade Federal de Minas Gerais - UFMG - Belo Horizonte (MG), Brasil.

${ }^{1}$ Programa de Pós-graduação em Ciências Fonoaudiológicas (Mestrado), Universidade Federal de Minas Gerais - UFMG - Belo Horizonte (MG), Brasil.

${ }^{2}$ Programa de Neurociências, Universidade Federal de Minas Gerais - UFMG - Belo Horizonte (MG), Brasil.

${ }^{3}$ Programa de Pós-graduação em Ciências Fonoaudiológicas, Departamento de Fonoaudiologia, Faculdade de Medicina, Universidade Federal de Minas Gerais - UFMG - Belo Horizonte (MG), Brasil.

Conflito de interesses: Não.

Contribuição dos autores: CAS foi responsável pela coleta e análise dos dados, redação do manuscrito e aprovação da versão final; AGE participou da orientação do trabalho, coleta e análise dos dados, redação do manuscrito, aprovação da versão final; SMAL foi responsável pela concepção do estudo e orientação de todas as etapas do trabalho, análise dos dados, redação do manuscrito e aprovação da versão final.

Financiamento: Edital - Chamada MCTIC/CNPq No 28/2018 - Universal Processo: 422625/2018; Edital - Chamada 01/2018 - Demanda Universal/ FAPEMIG - Processo: APQ-01354-18; Coordenação de Aperfeiçoamento de Pessoal de Nível Superior (CAPES) - Bolsa do Programa de Demanda Social; Bolsas de Produtividade em Pesquisa - PQ Processo: 308647/2018-1; Produtividade em Pesquisa CNPq-PQ; 308647/2018-1. O presente trabalho foi realizado com apoio da Coordenação de Aperfeiçoamento de Pessoal de Nível Superior - Brasil (CAPES) - Código de Financiamento 001.

Autor correspondente: Cintia Alves de Souza. E-mail: cintiasouzafono@gmail.com

Recebido: Março 02, 2021; Aceito: Novembro 05, 2021 


\section{INTRODUÇÃO}

A leitura é um processo complexo, que ativa diversos processos cognitivos que se inter-relacionam, a saber: a capacidade de processar, armazenar e recuperar informações; a habilidade de memória, de atenção, de raciocínio, de lógica e de processamento auditivo e visual ${ }^{(1,2)}$. Nesse contexto, estão presentes, também, os processos básicos de leitura, como o reconhecimento, que é a decodificação de palavras, e a extração do significado das palavras impressas. Ressalta-se, entretanto, que, mesmo sendo requisitos necessários, tais processos não são suficientes para que a compreensão ocorra ${ }^{(3,4)}$. Além disso, para que a leitura seja de fato efetiva é imprescindível que o leitor compreenda o material lido e seja capaz de utilizar os conhecimentos adquiridos posteriormente ${ }^{(5)}$.

Fica notória, portanto, a importância da leitura no processo de alfabetização, uma vez que ela se constitui a base para a aprendizagem de muitas áreas do conhecimento ${ }^{(5)}$. Assim, o fracasso escolar, ou seja, o desempenho não satisfatório no aprendizado das disciplinas é acarretado, especialmente, por dificuldades na leitura e na escrita ${ }^{(4)}$. Dessa forma, justifica-se o estudo da leitura à luz da presença de queixas de dificuldades escolares.

Dentre os fatores que podem influenciar a aprendizagem das habilidades escolares e o desenvolvimento da leitura, podese citar a motivação escolar. Estudo brasileiro ${ }^{(6)}$ evidenciou a contribuição relevante da motivação para a compreensão da leitura em alunos do ensino fundamental, justamente porque aspectos cognitivos e motivacionais interagem para produzir sucesso em tarefas acadêmicas. Assim, é pertinente afirmar que "construir significado durante a leitura é um ato motivado"(6:55).

Nesse contexto, o processamento das informações auditivas também desempenha um papel fundamental. O processamento auditivo é a capacidade do sistema nervoso central (SNC) de utilizar as informações auditivas de forma eficiente e efetiva ${ }^{(7)}$ e engloba diferentes habilidades.

A presente pesquisa foi pautada na habilidade auditiva de ordenação temporal, que se refere ao processamento de diversos estímulos auditivos, na ordem em que ocorrem ${ }^{(8)}$. Assim, é esperado que a pessoa consiga identificar a ordem exata de apresentação dos sons ${ }^{(8)}$. Essa habilidade, em conjunto com a resolução temporal, desempenha um importante papel na percepção e compreensão da fala e, por este motivo, ambas são pré-requisitos para o desenvolvimento de habilidades linguísticas ${ }^{(9)}$. Assim, alterações na percepção auditiva podem culminar em dificuldades ortográficas e na codificação/decodificação tanto de palavras, como de frases ${ }^{(9)}$.

Os testes de processamento auditivo são utilizados constantemente para averiguar a associação entre dificuldades escolares e alterações no desenvolvimento de habilidades auditivas ${ }^{(9)}$. Por conseguinte, indivíduos com queixas de dificuldades escolares comumente apresentam pior desempenho na avaliação do processamento auditivo, resultado explicado pelo atraso na maturação das habilidades auditivas, que são primordiais para a aprendizagem da leitura e da escrita ${ }^{(10)}$.

Por conseguinte, é evidente a importância do estudo da leitura, associado com as habilidades auditivas e motivação. A compreensão da relação entre esses aspectos contribuirá para avanços na prática da fonoaudiologia clínica, permitindo a elaboração e construção de melhores estratégias para atender o público com dificuldades de aprendizagem. Sendo assim, o objetivo do presente estudo foi verificar a associação entre o desempenho em leitura de crianças e adolescentes e a presença de queixas escolares, habilidade auditiva de ordenação temporal e motivação escolar.

\section{MÉTODOS}

Trata-se de estudo observacional analítico transversal, com amostra de conveniência composta por 36 participantes, na faixa etária de 9 a 12 anos. Para compor a amostra, foram realizadas duas diferentes abordagens: convite aos responsáveis pelas crianças atendidas no Ambulatório de Fonoaudiologia do Hospital das Clínicas da UFMG (HC-UFMG) e divulgação da pesquisa por meio de redes sociais.

O estudo foi aprovado pelo Comitê de Ética em Pesquisa da Universidade Federal de Minas Gerais - UFMG, sob o parecer 2.093.022. Os responsáveis assinaram o Termo de Consentimento Livre e Esclarecido - TCLE e os participantes assinaram o Termo de Assentimento Livre e Esclarecido (TALE).

Os critérios para inclusão dos participantes foram: ter idade entre 9 e 12 anos e estar regularmente matriculado em alguma instituição de ensino, independentemente de apresentar, ou não, queixas escolares. Foram considerados critérios de exclusão: apresentar perda auditiva ou queixa de alterações cognitivas, neurológicas, neuropsiquiátricas ou motoras, que impedissem a realização das avaliações; desistir durante a aplicação dos testes.

Os procedimentos da pesquisa foram:

- Questionário de caracterização sociodemográfica (idade e sexo) e escolar (tipo de financiamento da escola, ano escolar e presença de queixa de dificuldades escolares);

- Critério de Classificação Econômica do Brasil (CCEB $2018)^{(11)}$, que classifica os participantes em classes de A (maior poder aquisitivo) a E (menor poder aquisitivo), de acordo com o nível de instrução do chefe da família, a posse de bens materiais, as condições da rua e a origem da água utilizada no domicílio;

- Avaliação auditiva, que constou de audiometria tonal e timpanometria, a fim de verificar a integridade do sistema auditivo ${ }^{(12,13) ;}$

- Avaliação da habilidade auditiva de ordenação temporal simples $^{(14)}$ por meio dos Testes de Memória para Sons Verbais (TMSV) e Não Verbais (TMSNV) em Sequência. No TMSV, foi solicitado à criança que repetisse, da maneira que ouviu, as três sequências em que as sílabas /pa/,/ta/, /ca/, /fa/ foram apresentadas pelo examinador ${ }^{(14)}$. Já no TMSNV, foi solicitado que a criança apontasse a ordem correta das três sequências em que os quatro instrumentos (sino, guizo, coco, agogô) foram apresentados ${ }^{(14)}$. Os testes foram realizados sem pista visual;

- Avaliação da habilidade auditiva de ordenação temporal complexa ${ }^{(14)}$, por meio dos Testes de Padrão de Frequência (TPF) e de Padrão de Duração (TPD), realizados em escuta diótica. No TPF, foram apresentadas aos participantes sequências de três e quatro sons, com diferentes combinações dos estímulos de frequência baixa $(440 \mathrm{~Hz})$ e alta $(493 \mathrm{~Hz})$. No TPD, também foram apresentadas sequências de três e quatro sons, dessa vez com diferentes combinações de sons de frequência 
fixa $(440 \mathrm{~Hz})$ de longa duração $(2000 \mathrm{~ms})$ e de curta duração $(500 \mathrm{~ms})$. A marcação foi realizada pelo próprio participante, da seguinte maneira: X para os sons agudos/ finos, $\mathrm{O}$ para os sons graves/grossos, traço comprido para os sons longos e ponto para os sons curtos ${ }^{(15-17) ;}$

- Teste de Desempenho Escolar - TDE(18), aplicado para avaliar as habilidades de escrita, leitura e aritmética, com seus respectivos subtestes. A classificação foi realizada a partir dos escores brutos de cada subteste e do escore bruto total, de acordo com o ano escolar. Foram considerados como "bom desempenho escolar" os escores classificados como superiores e médios e como "mau desempenho escolar", os escores classificados como inferiores;

- Provas de Avaliação dos Processos de Leitura-PROLEC ${ }^{(19)}$, em aplicação na versão reduzida, composta pelas seguintes provas: prova 2 - Igual-diferente em palavras e pseudopalavras; prova 5 - Leitura de pseudopalavras; prova 7 - Estruturas gramaticais; prova 10 - Compreensão de textos. As pontuações foram obtidas por prova e os resultados interpretados de acordo com o critério de referência para o ano escolar do participante ${ }^{(19) ;}$

- Escala para Avaliação da Motivação Escolar Infantojuvenil - EAME-IJ(20), que mensura as fontes de motivação (intrínseca e extrínseca), bem como sua intensidade. A escala foi lida pelo aplicador para que o participante respondesse oralmente. Considerando-se a pontuação da motivação intrínseca, extrínseca e a somatória geral, os resultados foram classificados em motivação baixa, média ou alta, de acordo com a escolaridade do examinado.

A coleta dos dados foi realizada no Observatório de Saúde Funcional em Fonoaudiologia (OSF) da Faculdade de Medicina da UFMG. Os testes foram aplicados em sessões individuais, sendo necessárias duas sessões, com duração de uma hora cada. Os testes auditivos foram aplicados em uma sala tratada acusticamente e os demais, em sala com menor exposição ao ruído.

Considerou-se como variável resposta deste estudo "os processos de leitura", que foram avaliados pelas Provas de Avaliação dos Processos de Leitura - PROLEC ${ }^{(19)}$. As variáveis explicativas foram: habilidade auditiva de ordenação temporal, dados sociodemográficos (sexo, idade, ano escolar e classificação socioeconômica) e aspectos escolares (ano em curso, desempenho e motivação).

Os dados coletados foram digitados, conferidos e categorizados em um banco de dados. Foram realizadas análises descritivas da distribuição de frequência absoluta e relativa de todas as variáveis categóricas e análise das medidas de tendência central e de dispersão das variáveis contínuas. Para as análises de associação, foram realizados os seguintes agrupamentos: os domínios do TDE foram divididos em duas categorias: "desempenho escolar inferior" e "desempenho escolar médio/ superior", segundo a soma total dos subtestes; as classes socioeconômicas do CCEB foram agrupadas em classes A/B e classes C/D-E; os resultados do PROLEC foram reorganizados em "normais" e "alterados", sendo que os resultados alterados englobaram os desempenhos classificados como "dificuldade" e "dificuldade grande" e os resultados dos testes TMSV e TMSNV, TPD e TPF (três e quatro sons) foram categorizados em "adequados" e "inadequados", de acordo com os critérios de referência previstos na literatura ${ }^{(14)}$. O teste Qui-quadrado de Pearson e o teste Exato de Fisher foram utilizados para as análises de associação, considerando como estatisticamente significativas as que apresentaram valor de $\mathrm{p} \leq 0,05$.

Para entrada e processamento dos dados foi utilizado o software SPSS, versão 21.0.

\section{RESULTADOS}

Nesta pesquisa, a amostra foi composta por 36 participantes, sendo a maioria $(61,1 \%)$ do sexo masculino. A faixa etária variou de 9 a 12 anos, 11 meses e 29 dias, com média de idade de 10 anos e 2 meses e mediana de 10 anos (Figura 1).

Em relação à escolaridade, a amostra foi distribuída entre o terceiro e o sétimo ano do ensino fundamental, sendo que a maior parte cursava o sexto ano $(30,6 \%)$, estava matriculada em instituições de ensino de financiamento público $(88,9 \%)$, apresentava desempenho escolar inferior $(80,6 \%)$ e apenas $19,4 \%$ não apresentaram queixa de dificuldades escolares (Figuras 1 e 2).

No que diz respeito à classificação socioeconômica, $52,8 \%$ da amostra se concentraram nas classes C e D-E. Os responsáveis por um participante não responderam ao CCEB e, por isso, a amostra desse questionário totalizou 35 participantes (Figura 1).

No que se refere à motivação escolar, $50,0 \%(\mathrm{n}=18)$ apresentaram motivação intrínseca baixa, 44,4\% ( $\mathrm{n}=16)$ apresentaram motivação extrínseca média e $50,0 \%(n=18)$ apresentaram motivação geral média (Figura 2).

As Provas de Avaliação dos Processos de Leitura - PROLEC foram aplicadas em 36 participantes e verificou-se que o desempenho dentro dos padrões de normalidade, em cada prova, foi de $52,8 \%$ na prova 2 (Igual-diferente em palavras e pseudopalavras); $61,1 \%$ na prova 5 (Leitura de pseudopalavras); $66,7 \%$ na prova 7 (Estruturas gramaticais) e $50,0 \%$ na prova 10 (Compreensão de textos).

Além disso, $75,0 \%$ dos participantes apresentaram adequação da ordenação temporal simples para sons verbais e $86,1 \%$ para sons não verbais em sequência.

No TPF, 8 participantes $(22,2 \%)$ apresentaram resultado adequado na sequência de 3 sons, ao passo que $10(27,8 \%)$ obtiveram adequação na sequência de 4 sons. Já no TPD, 9 crianças e adolescentes $(25,0 \%)$ apresentaram adequação das respostas na sequência de 3 sons, enquanto 21 (58,3\%) obtiveram adequação na sequência de 4 sons. Assim, considerando-se os resultados obtidos na avaliação da ordenação temporal complexa, pode-se afirmar que grande parte dos participantes obteve resultado inadequado.

$\mathrm{Na}$ análise de associação de todas as provas do PROLEC com o sexo, verificou-se que os participantes do sexo masculino apresentaram maior porcentagem de dificuldade quando comparado ao sexo feminino. Na análise da associação do PROLEC com a idade, observou-se que nas provas 2 (Igual-diferente em palavras e pseudopalavras), 5 (Leitura de pseudopalavras) e 10 (Compreensão de textos) o maior número de alterações concentrou-se na idade de 9 anos, sendo que, na prova 7 (Estruturas gramaticais), a distribuição dos participantes que apresentaram dificuldades foi similar nas idades de 9 e 11 anos. Contudo, cabe ressaltar que essas associações não apresentaram significância estatística (Tabela 1). 

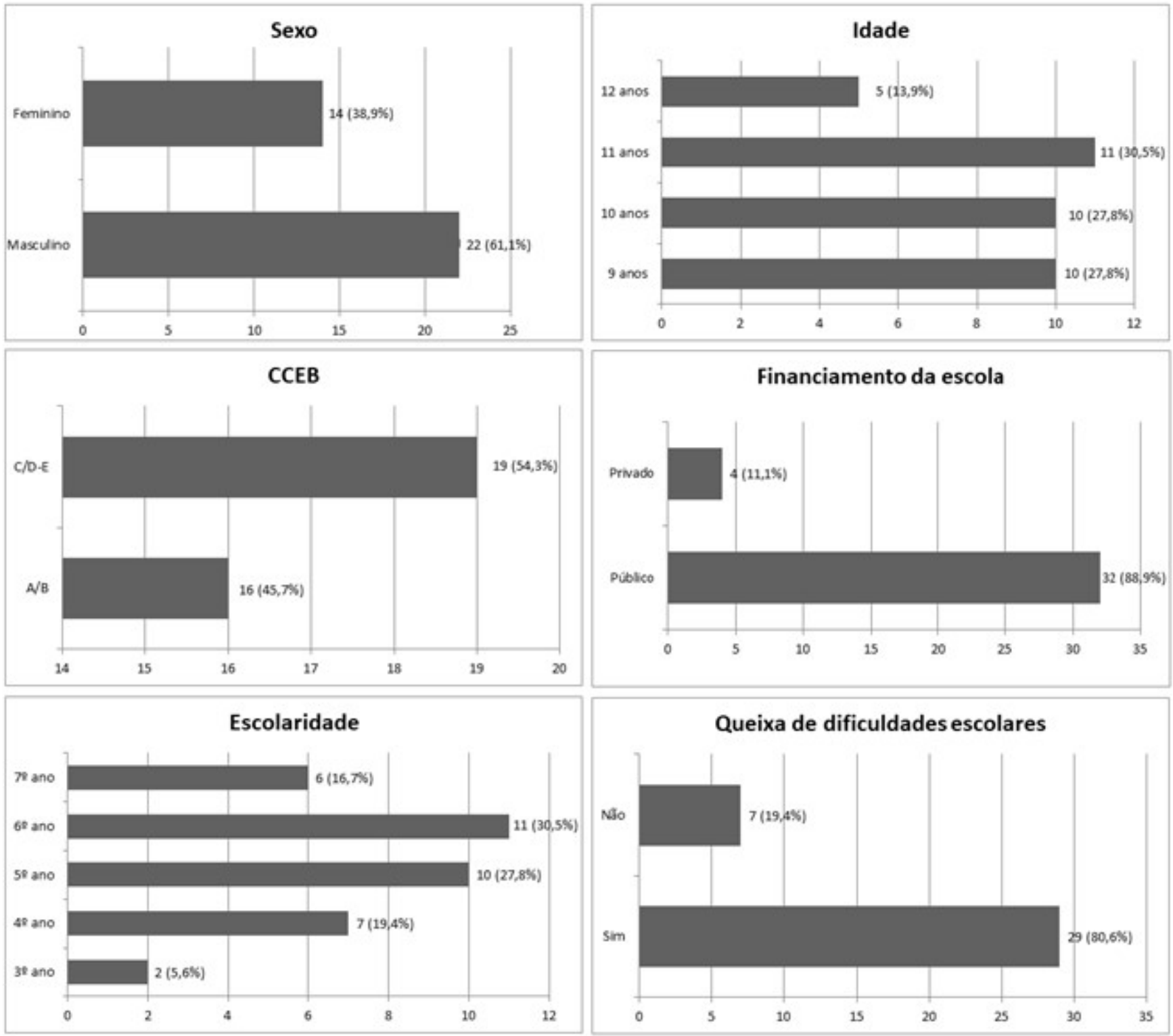

Figura 1. Gráficos de caracterização sociodemográfica, socioeconômica e escolar da amostra Legenda: $C C E B$ = Critério de Classificação Econômica do Brasil; A/B, C/D-E = classes econômicas

Por fim, na associação da classificação socioeconômica com o PROLEC, os participantes que apresentaram dificuldades nas provas pertenciam, em grande parte, às classes C/D-E (Tabela 1).

Não houve significância estatística na análise de associação da escolaridade com o PROLEC. Entretanto, foi possível verificar que a maior parte dos resultados inadequados foi proveniente de alunos da rede pública de ensino, com queixa de dificuldades escolares e baixo desempenho acadêmico. Além disso, observou-se significância estatística na associação da prova 7 (Estruturas gramaticais) com a motivação escolar geral, bem como na associação das provas 2 (Igual-diferente em palavras e pseudopalavras) e 7 (Estruturas gramaticais) com o TDE leitura. (Tabela 2).

Verificou-se, na associação entre PROLEC e ordenação temporal simples, que a maioria dos participantes com alteração nas tarefas de leitura apresentou adequação das habilidades auditivas temporais. Cabe salientar que a única associação que apresentou significância estatística foi entre a prova 5 (Leitura de pseudopalavras) e o TMSNV (Tabela 3).

$\mathrm{Na}$ associação entre PROLEC e ordenação temporal complexa, observou-se que quase a totalidade dos participantes com dificuldades nas tarefas de leitura também apresentou inadequação nos testes de Padrão de Frequência e de Padrão de Duração, tanto nas sequências com 3 sons, quanto com 4 sons. A única exceção foi no TPD (4 sons), na qual a amostra se dividiu de forma similar: metade dos participantes com resultado alterado na prova 10 (Compreensão de textos) apresentou resultado adequado no TPD (4 sons) e, a outra metade, apresentou resultado inadequado no TPD (4 sons) (Tabela 4).

A associação das provas 2 (Igual-diferente em palavras e pseudopalavras) e 5 (Leitura de pseudopalavras) com o TPF (três sons) e com o TPD (quatro sons) apresentou significância estatística (Tabela 4). 

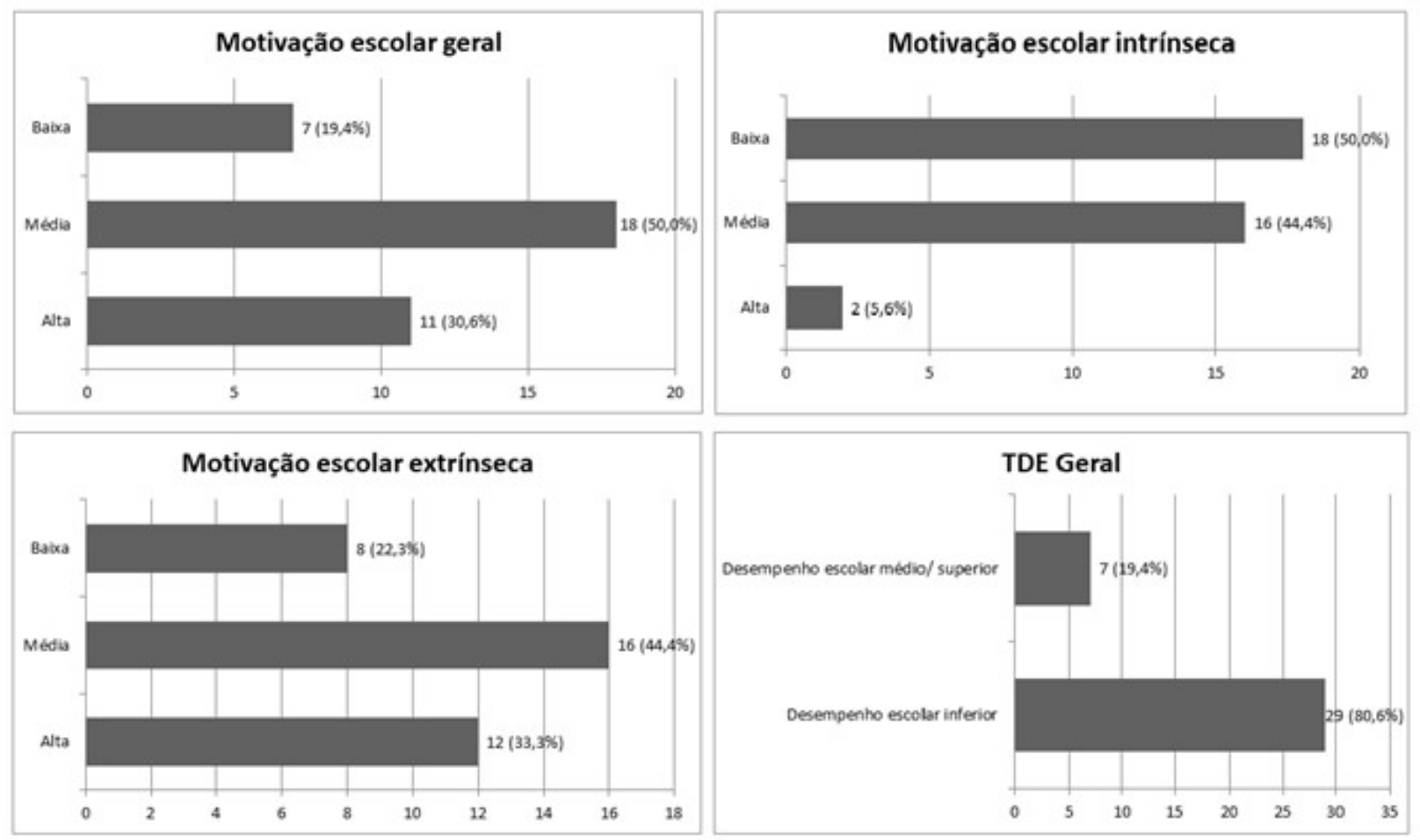

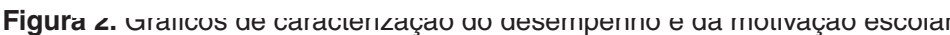

Legenda: TDE = Teste de Desempenho Escolar

Tabela 1. Associação entre os resultados das Provas de Avaliação dos Processos de Leitura e as variáveis sociodemográficas e socioeconômicas

\begin{tabular}{|c|c|c|c|c|c|c|c|c|c|}
\hline \multirow{3}{*}{ PROLEC } & & \multicolumn{4}{|c|}{ Idade } & \multicolumn{2}{|c|}{ Sexo } & \multicolumn{2}{|c|}{ CCEB } \\
\hline & & 9 & 10 & 11 & 12 & $\mathbf{F}$ & M & A/B & C/D-E \\
\hline & & N (\%) & N (\%) & $\mathbf{N}(\%)$ & N (\%) & N (\%) & N (\%) & $\mathbf{N}(\%)$ & N (\%) \\
\hline \multirow{4}{*}{$\begin{array}{l}\text { Prova } 2 \\
\text { Igual-diferente } \\
\text { em palavras e } \\
\text { pseudopalavras }\end{array}$} & Normal & $2(10,5)$ & $7(36,9)$ & $8(42,1)$ & $2(10,5)$ & $9(47,4)$ & $10(52,6)$ & $9(50,0)$ & $9(50,0)$ \\
\hline & Alterado & $8(47,2)$ & $3(17,6)$ & $3(17,6)$ & $3(17,6)$ & $5(29,4)$ & $12(70,6)$ & $7(41,2)$ & $10(58,8)$ \\
\hline & Total & $10(27,8)$ & $10(27,8)$ & $11(30,6)$ & $5(13,8)$ & $14(38,9)$ & $22(61,1)$ & $16(45,7)$ & $19(54,3)$ \\
\hline & Valor de $p$ & \multicolumn{4}{|c|}{$0,055^{*}$} & \multicolumn{2}{|c|}{0,270 * } & \multicolumn{2}{|c|}{0,600 * } \\
\hline \multirow{4}{*}{$\begin{array}{l}\text { Prova } 5 \\
\text { Leitura de } \\
\text { pseudopalavras }\end{array}$} & Normal & $5(22,7)$ & $7(31,8)$ & $7(31,8)$ & $3(13,7)$ & $9(40,9)$ & $13(59,1)$ & $12(57,1)$ & $9(42,9)$ \\
\hline & Alterado & $5(35,7)$ & $3(21,4)$ & $4(28,6)$ & $2(14,3)$ & $5(35,7)$ & $9(64,3)$ & $4(28,6)$ & $10(71,4)$ \\
\hline & Total & $10(27,8)$ & $10(27,8)$ & $11(30,6)$ & $5(13,8)$ & $14(38,9)$ & $22(61,1)$ & $16(45,7)$ & $19(54,3)$ \\
\hline & Valor de $p$ & \multicolumn{4}{|c|}{0,829 * } & 0,755 * & & 0,096 * & \\
\hline \multirow{4}{*}{$\begin{array}{l}\text { Prova } 7 \\
\text { Estruturas } \\
\text { gramaticais }\end{array}$} & Normal & $6(25,0)$ & $7(29,2)$ & $7(29,2)$ & $4(16,6)$ & $11(45,8)$ & $13(54,2)$ & $13(56,5)$ & $10(43,5)$ \\
\hline & Alterado & $4(33,3)$ & $3(25,0)$ & $4(33,3)$ & $1(8,4)$ & $3(25,0)$ & $9(75,0)$ & $3(25,0)$ & $9(75,0)$ \\
\hline & Total & $10(27,8)$ & $10(27,8)$ & $11(30,6)$ & $5(13,8)$ & $14(38,9)$ & $22(61,1)$ & $16(45,7)$ & $19(54,3)$ \\
\hline & Valor de $p$ & \multicolumn{4}{|c|}{0,874 * } & 0,292 ** & & 0,152 ** & \\
\hline \multirow{4}{*}{$\begin{array}{l}\text { Prova } 10 \\
\text { Compreensão } \\
\text { de textos }\end{array}$} & Normal & $4(22,2)$ & $5(27,8)$ & $7(38,9)$ & $2(11,1)$ & $9(50,0)$ & $9(50,0)$ & $9(52,9)$ & $8(47,1)$ \\
\hline & Alterado & $6(33,3)$ & $5(27,8)$ & $4(22,2)$ & $3(16,7)$ & $5(27,8)$ & $13(72,2)$ & $7(38,9)$ & $11(61,1)$ \\
\hline & Total & $10(27,8)$ & $10(27,8)$ & $11(30,6)$ & $5(13,8)$ & $14(38,9)$ & $22(61,1)$ & $16(45,7)$ & $19(54,3)$ \\
\hline & Valor de $p$ & \multicolumn{4}{|c|}{0,701 * } & \multicolumn{2}{|c|}{$0,171^{*}$} & \multicolumn{2}{|c|}{$0,404^{*}$} \\
\hline
\end{tabular}

${ }^{*}$ Teste Qui-quadrado de Pearson; ${ }^{* *}$ Teste Exato de Fisher

Legenda: $\mathrm{PROLEC}=$ Provas de Avaliação dos Processos de Leitura; $\mathrm{F}=$ Feminino; $\mathrm{M}=$ Masculino; $\mathrm{CCEB}=$ Critério de Classificação Econômica do Brasil; $\mathrm{N}=$ número de participantes; \% = percentual; A/B, C/D-E = classes econômicas

\section{DISCUSSÃO}

Em relação à variável resposta, a maior porcentagem de resultados inadequados no PROLEC, que ocorreu na prova 10 (Compreensão de textos), seguida pelas provas 2
(Igual-diferente em palavras e pseudopalavras), 5 (Leitura de pseudopalavras) e 7 (Estruturas gramaticais), pôde indicar que o desempenho no processo semântico (compreensão de textos) é comprometido pela dificuldade apresentada nos processos de identificação de letras (igual-diferente em palavras e pseudopalavras), léxico (leitura de pseudopalavras) e sintático 
Tabela 2. Associação entre os resultados das Provas de Avaliação dos Processos de Leitura e as variáveis escolares

\begin{tabular}{|c|c|c|c|c|c|c|c|c|c|c|c|c|c|c|c|}
\hline \multirow{2}{*}{\multicolumn{2}{|c|}{ PROLEC }} & \multicolumn{5}{|c|}{ Ano escolar } & \multicolumn{2}{|c|}{ Tipo de escola } & \multicolumn{2}{|c|}{$\begin{array}{l}\text { Queixa } \\
\text { escolar }\end{array}$} & \multicolumn{2}{|c|}{ TDE leitura } & \multicolumn{3}{|c|}{$\begin{array}{c}\text { Motivação escolar } \\
\text { geral }\end{array}$} \\
\hline & & $\frac{3^{\circ}}{N(\%)}$ & $\frac{4^{\circ}}{N(\%)}$ & $\frac{5^{\circ}}{N(\%)}$ & $\frac{6^{\circ}}{N(\%)}$ & $\frac{7^{\circ}}{N(\%)}$ & $\frac{\text { Privada }}{\mathrm{N}(\%)}$ & $\frac{\text { Pública }}{\mathrm{N}(\%)}$ & $\frac{\operatorname{Sim}}{\mathrm{N}(\%)}$ & $\frac{\text { Não }}{\mathrm{N}(\%)}$ & $\frac{\text { DEMS }}{\mathrm{N}(\%)}$ & $\frac{\text { DEI }}{\mathrm{N}(\%)}$ & $\frac{\text { Alta }}{\mathrm{N}(\%)}$ & $\frac{\text { Média }}{\mathrm{N}(\%)}$ & $\frac{\text { Baixa }}{\mathrm{N}(\%)}$ \\
\hline \multirow{4}{*}{$\begin{array}{l}\text { Prova } 2 \\
\text { Igual- } \\
\text { diferente em } \\
\text { palavras e } \\
\text { pseudopalavras }\end{array}$} & Normal & $0(0,0)$ & $2(10,5)$ & $7(36,8)$ & $7(36,8)$ & $\begin{array}{c}3 \\
(15,9)\end{array}$ & $3(15,8)$ & $\begin{array}{c}16 \\
(84,2)\end{array}$ & $\begin{array}{c}13 \\
(68,4)\end{array}$ & $\begin{array}{c}6 \\
(31,6)\end{array}$ & $8(42,1)$ & $\begin{array}{c}11 \\
(57,9)\end{array}$ & $6(31,6)$ & $\begin{array}{c}11 \\
(57,9)\end{array}$ & $2(10,5)$ \\
\hline & Alterado & $2(11,8)$ & $5(29,5)$ & $3(17,6)$ & $4(23,5)$ & $3(17,6)$ & $1(5,9)$ & $\begin{array}{c}16 \\
(94,1)\end{array}$ & $\begin{array}{c}16 \\
(94,1)\end{array}$ & $1(5,9)$ & $1(5,9)$ & $\begin{array}{c}16 \\
(94,1)\end{array}$ & $5(29,4)$ & $7(41,2)$ & $5(29,4)$ \\
\hline & Total & $2(5,6)$ & $7(19,4)$ & $\begin{array}{c}10 \\
(27,8)\end{array}$ & $\begin{array}{c}11 \\
(30,6)\end{array}$ & $\begin{array}{c}6 \\
(16,6)\end{array}$ & $4(11,1)$ & $\begin{array}{c}32 \\
(88,9)\end{array}$ & $\begin{array}{c}29 \\
(80,6)\end{array}$ & $\begin{array}{c}7 \\
(19,4)\end{array}$ & $9(25,0)$ & $\begin{array}{c}27 \\
(75,0)\end{array}$ & $\begin{array}{c}11 \\
(30,6)\end{array}$ & $\begin{array}{c}18 \\
(50,0)\end{array}$ & $7(19,4)$ \\
\hline & $\begin{array}{l}\text { Valor } \\
\text { de } p\end{array}$ & & & \multicolumn{2}{|l|}{0,230 * } & & \multicolumn{2}{|c|}{$0,605^{* *}$} & \multicolumn{2}{|c|}{$0,092^{* *}$} & \multicolumn{2}{|c|}{0,020 ** } & & 0,339 * & \\
\hline \multirow{4}{*}{$\begin{array}{l}\text { Prova } 5 \\
\text { Leitura de } \\
\text { pseudopalavras }\end{array}$} & Normal & $0(0,0)$ & $4(18,2)$ & $7(31,8)$ & $7(31,8)$ & $\begin{array}{c}4 \\
(18,2)\end{array}$ & $4(18,2)$ & $\begin{array}{c}18 \\
(81,8)\end{array}$ & $\begin{array}{c}16 \\
(72,7)\end{array}$ & $\begin{array}{c}6 \\
(27,3)\end{array}$ & $7(31,8)$ & $\begin{array}{c}15 \\
(68,2)\end{array}$ & $9(40,9)$ & $\begin{array}{c}10 \\
(45,5)\end{array}$ & $3(13,6)$ \\
\hline & Alterado & $2(14,3)$ & $3(21,4)$ & $3(21,4)$ & $4(28,6)$ & $\begin{array}{c}2 \\
(14,3)\end{array}$ & $0(0,0)$ & $\begin{array}{c}14 \\
(100,0)\end{array}$ & $\begin{array}{c}13 \\
(92,9)\end{array}$ & $1(7,1)$ & $2(14,3)$ & $\begin{array}{c}12 \\
(85,7)\end{array}$ & $2(14,3)$ & $8(57,1)$ & $4(28,6)$ \\
\hline & Total & $2(5,6)$ & $7(19,4)$ & $\begin{array}{c}10 \\
(27,8)\end{array}$ & $\begin{array}{c}11 \\
(30,6)\end{array}$ & $\begin{array}{c}6 \\
(16,6)\end{array}$ & $4(11,1)$ & $\begin{array}{c}32 \\
(88,9)\end{array}$ & $\begin{array}{c}29 \\
(80,6)\end{array}$ & $\begin{array}{c}7 \\
(19,4)\end{array}$ & $9(25,0)$ & $\begin{array}{c}27 \\
(75,0)\end{array}$ & $\begin{array}{c}11 \\
(30,6)\end{array}$ & $\begin{array}{c}18 \\
(50,0)\end{array}$ & $7(19,4)$ \\
\hline & $\begin{array}{l}\text { Valor } \\
\text { de } p\end{array}$ & & & \multicolumn{2}{|l|}{0,459 * } & & \multicolumn{2}{|c|}{0,141 ** } & \multicolumn{2}{|c|}{0,209 ** } & \multicolumn{2}{|c|}{0,432 ** } & & 0,202 * & \\
\hline \multirow{4}{*}{$\begin{array}{l}\text { Prova } 7 \\
\text { Estruturas } \\
\text { gramaticais }\end{array}$} & Normal & $0(0,0)$ & $5(20,8)$ & $7(29,2)$ & $8(33,3)$ & $\begin{array}{c}4 \\
(16,7)\end{array}$ & $4(16,7)$ & $\begin{array}{c}20 \\
(83,3)\end{array}$ & $18(75,0)$ & $\begin{array}{c}6 \\
(25,0)\end{array}$ & $9(37,5)$ & $\begin{array}{c}15 \\
(62,5)\end{array}$ & $7(29,2)$ & $\begin{array}{c}15 \\
(62,5)\end{array}$ & $2(8,3)$ \\
\hline & Alterado & $2(16,7)$ & $2(16,7)$ & $3(25,0)$ & $3(25,0)$ & $\begin{array}{c}2 \\
(16,7)\end{array}$ & $0(0,0)$ & $\begin{array}{c}12 \\
(100,0)\end{array}$ & $\begin{array}{c}11 \\
(91,7)\end{array}$ & $1(8,3)$ & $0(0,0)$ & $\begin{array}{c}12 \\
(100,0)\end{array}$ & $4(33,3)$ & $3(25,0)$ & $5(41,7)$ \\
\hline & Total & $2(5,6)$ & $7(19,4)$ & $\begin{array}{c}10 \\
(27,8)\end{array}$ & $\begin{array}{c}11 \\
(30,6)\end{array}$ & $\begin{array}{c}6 \\
(16,6)\end{array}$ & $4(11,1)$ & $\begin{array}{c}32 \\
(88,9)\end{array}$ & $\begin{array}{c}29 \\
(80,6)\end{array}$ & $7(19,4)$ & $9(25,0)$ & $\begin{array}{c}27 \\
(75,0)\end{array}$ & $\begin{array}{c}11 \\
(30,6)\end{array}$ & $\begin{array}{c}18 \\
(50,0)\end{array}$ & $7(19,4)$ \\
\hline & $\begin{array}{l}\text { Valor } \\
\text { de } p\end{array}$ & & & \multicolumn{2}{|l|}{0,367 * } & & 0,27 & 78 ** & 0,3 & $34^{* *}$ & 0,01 & 6 ** & & 0,032 * & \\
\hline $\begin{array}{l}\text { Prova } 10 \\
\text { Compreensão }\end{array}$ & Normal & $0(0,0)$ & $3(16,7)$ & $6(33,3)$ & $7(38,9)$ & $\begin{array}{c}2 \\
(11,1)\end{array}$ & $3(16,7)$ & $\begin{array}{c}15 \\
(83,3)\end{array}$ & $\begin{array}{c}14 \\
(77,8)\end{array}$ & $\begin{array}{c}4 \\
(22,2)\end{array}$ & $5(27,8)$ & $\begin{array}{c}13 \\
(72,2)\end{array}$ & $6(33,3)$ & $9(50,0)$ & $3(16,7)$ \\
\hline & Alterado & $2(11,2)$ & $4(22,2)$ & $4(22,2)$ & $4(22,2)$ & $\begin{array}{c}4 \\
(22,2)\end{array}$ & $1(5,6)$ & $\begin{array}{c}17 \\
(94,4)\end{array}$ & $\begin{array}{c}15 \\
(83,3)\end{array}$ & $\begin{array}{c}3 \\
(16,7)\end{array}$ & $4(22,2)$ & $\begin{array}{c}14 \\
(77,8)\end{array}$ & $5(27,8)$ & $9(50,0)$ & $4(22,2)$ \\
\hline & Total & $2(5,6)$ & $7(19,4)$ & $\begin{array}{c}10 \\
(27,8)\end{array}$ & $\begin{array}{c}11 \\
(30,6)\end{array}$ & $\begin{array}{c}6 \\
(16,6)\end{array}$ & $4(11,1)$ & $\begin{array}{c}32 \\
(88,9)\end{array}$ & $\begin{array}{c}29 \\
(80,6)\end{array}$ & $\begin{array}{c}7 \\
(19,4)\end{array}$ & $9(25,0)$ & $\begin{array}{c}27 \\
(75,0)\end{array}$ & $\begin{array}{c}11 \\
(30,6)\end{array}$ & $\begin{array}{c}18 \\
(50,0)\end{array}$ & $7(19,4)$ \\
\hline & $\begin{array}{l}\text { Valor } \\
\text { de } p\end{array}$ & & & 0,402 * & & & 0,60 & $03^{\text {** }}$ & & & 0,70 & 00 * & & 0,890 * & \\
\hline
\end{tabular}

*Teste Qui-quadrado de Pearson; **Teste Exato de Fisher

Legenda: PROLEC = Provas de Avaliação dos Processos de Leitura; TDE leitura = Teste de Desempenho Escolar - subteste de leitura; DEI = Desempenho escolar inferior; DEMS = Desempenho escolar médio/superior; $\mathrm{N}$ = número de participantes; \% = percentual

Tabela 3. Associação entre os resultados das Provas de Avaliação dos Processos de Leitura e a ordenação temporal simples

\begin{tabular}{|c|c|c|c|c|c|}
\hline \multirow{2}{*}{ PROLEC } & & \multicolumn{2}{|c|}{ TMSV } & \multicolumn{2}{|c|}{ TMSNV } \\
\hline & & Adequado & Inadequado & Adequado & Inadequado \\
\hline \multirow{4}{*}{$\begin{array}{l}\text { Prova } 2 \\
\text { Igual-diferente } \\
\text { em palavras e } \\
\text { pseudopalavras }\end{array}$} & Normal & $16(84,2)$ & $3(15,8)$ & $16(84,2)$ & $3(15,8)$ \\
\hline & Alterado & $11(64,7)$ & $6(35,3)$ & $15(88,2)$ & $2(11,8)$ \\
\hline & Total & $27(75,0)$ & $9(25,0)$ & $31(86,1)$ & $5(13,9)$ \\
\hline & Valor de $p$ & & & & \\
\hline \multirow{4}{*}{$\begin{array}{l}\text { Prova } 5 \\
\text { Leitura de } \\
\text { pseudopalavras }\end{array}$} & Normal & $21(95,5)$ & $1(4,5)$ & $18(81,8)$ & $4(18,2)$ \\
\hline & Alterado & $6(42,9)$ & $8(57,1)$ & $13(92,9)$ & $1(7,1)$ \\
\hline & Total & $27(75,0)$ & $9(25,0)$ & $31(86,1)$ & $5(13,9)$ \\
\hline & Valor de $p$ & & & & \\
\hline \multirow{4}{*}{$\begin{array}{l}\text { Prova } 7 \\
\text { Estruturas } \\
\text { gramaticais }\end{array}$} & Normal & $20(83,3)$ & $4(16,7)$ & $20(83,3)$ & $4(16,7)$ \\
\hline & Alterado & $7(58,3)$ & $5(41,7)$ & $11(91,7)$ & $1(8,3)$ \\
\hline & Total & $27(75,0)$ & $9(25,0)$ & $31(86,1)$ & $5(13,9)$ \\
\hline & Valor de $p$ & & & & \\
\hline \multirow{4}{*}{$\begin{array}{l}\text { Prova } 10 \\
\text { Compreensão de } \\
\text { textos }\end{array}$} & Normal & $16(88,9)$ & $2(11,1)$ & $15(83,3)$ & $3(16,7)$ \\
\hline & Alterado & $11(61,1)$ & $7(38,9)$ & $16(88,9)$ & $2(11,1)$ \\
\hline & Total & $27(75,0)$ & $9(25,0)$ & $31(86,1)$ & $5(13,9)$ \\
\hline & Valor de $p$ & \multicolumn{2}{|c|}{0,121 ** } & \multicolumn{2}{|c|}{1,000 ** } \\
\hline
\end{tabular}

*Teste Qui-quadrado de Pearson; **Teste Exato de Fisher

Legenda: PROLEC = Provas de Avaliação dos Processos de Leitura; TMSV = Teste de Memória de Sons Verbais em Sequência; TMSNV = Teste de Memória de Sons Não Verbais em Sequência; $\mathrm{N}=$ número de participantes; \% = percentual 
Tabela 4. Associação entre os resultados das Provas de Avaliação dos Processos de Leitura e a ordenação temporal complexa

\begin{tabular}{|c|c|c|c|c|c|c|c|c|c|}
\hline \multirow[b]{2}{*}{ PROLEC } & & \multicolumn{2}{|c|}{ TPF 3 sons } & \multicolumn{2}{|c|}{ TPF 4 sons } & \multicolumn{2}{|c|}{ TPD 3 sons } & \multicolumn{2}{|c|}{ TPD 4 sons } \\
\hline & & Adequado & Inadequado & Adequado & Inadequado & Adequado & Inadequado & Adequado & Inadequado \\
\hline \multirow{4}{*}{$\begin{array}{l}\text { Prova } 2 \\
\text { Igual-diferente } \\
\text { em palavras e } \\
\text { pseudopalavras }\end{array}$} & Normal & $7(36,8)$ & $12(63,2)$ & $8(42,1)$ & $11(57,9)$ & $6(31,6)$ & $13(68,4)$ & $14(73,7)$ & $5(26,3)$ \\
\hline & Alterado & $1(5,9)$ & $16(94,1)$ & $2(11,8)$ & $15(88,2)$ & $3(17,6)$ & $14(82,4)$ & $7(41,2)$ & $10(58,8)$ \\
\hline & Total & $8(22,2)$ & $28(77,8)$ & $10(27,8)$ & $26(72,2)$ & $9(25,0)$ & $27(75,0)$ & $21(58,3)$ & $15(41,7)$ \\
\hline & Valor de $p$ & \multicolumn{2}{|c|}{0,044 ** } & \multicolumn{2}{|c|}{$0,065^{* *}$} & \multicolumn{2}{|c|}{$0,451^{* *}$} & \multicolumn{2}{|c|}{0,048 * } \\
\hline \multirow{4}{*}{$\begin{array}{l}\text { Prova } 5 \\
\text { Leitura de } \\
\text { pseudopalavras }\end{array}$} & Normal & $8(36,4)$ & $14(63,6)$ & $9(40,9)$ & $13(59,1)$ & $8(36,4)$ & $14(63,6)$ & $16(72,7)$ & $6(27,3)$ \\
\hline & Alterado & $0(0,0)$ & $14(100,0)$ & $1(7,1)$ & $13(92,9)$ & $1(7,1)$ & $13(92,9)$ & $5(35,7)$ & $9(64,3)$ \\
\hline & Total & $8(22,2)$ & $28(77,8)$ & $10(27,8)$ & $26(72,2)$ & $9(25,0)$ & $27(75,0)$ & $21(58,3)$ & $15(41,7)$ \\
\hline & Valor de $p$ & \multicolumn{2}{|c|}{0,013 ** } & \multicolumn{2}{|c|}{0,054 ** } & \multicolumn{2}{|c|}{$0,062^{* *}$} & \multicolumn{2}{|c|}{0,028 * } \\
\hline \multirow{4}{*}{$\begin{array}{l}\text { Prova } 7 \\
\text { Estruturas } \\
\text { gramaticais }\end{array}$} & Normal & $7(29,2)$ & $17(70,8)$ & $8(33,3)$ & $16(66,7)$ & $7(29,2)$ & $17(70,8)$ & $16(66,7)$ & $8(33,3)$ \\
\hline & Alterado & $1(8,3)$ & $11(91,7)$ & $2(16,7)$ & $10(83,3)$ & $2(16,7)$ & $10(83,3)$ & $5(41,7)$ & $7(58,3)$ \\
\hline & Total & $8(22,2)$ & $28(77,8)$ & $10(27,8)$ & $26(72,2)$ & $9(25,0)$ & $27(75,0)$ & $21(58,3)$ & $15(41,7)$ \\
\hline & Valor de $p$ & \multicolumn{2}{|c|}{$0,224^{\star *}$} & \multicolumn{2}{|c|}{$0,438^{* *}$} & \multicolumn{2}{|c|}{$0,685^{* *}$} & \multicolumn{2}{|c|}{0,151 * } \\
\hline \multirow{4}{*}{$\begin{array}{l}\text { Prova } 10 \\
\text { Compreensão de } \\
\text { textos }\end{array}$} & Normal & $5(27,8)$ & $13(72,2)$ & $7(38,9)$ & $11(61,1)$ & $7(38,9)$ & $11(61,1)$ & $12(66,7)$ & $6(33,3)$ \\
\hline & Alterado & $3(16,7)$ & $15(83,3)$ & $3(16,7)$ & $15(83,3)$ & $2(11,1)$ & $16(88,9)$ & $9(50,0)$ & $9(50,0)$ \\
\hline & Total & $8(22,2)$ & $28(77,8)$ & $10(27,8)$ & $26(72,2)$ & $9(25,0)$ & $27(75,0)$ & $21(58,3)$ & $15(41,7)$ \\
\hline & Valor de $p$ & \multicolumn{2}{|c|}{0,691 ** } & \multicolumn{2}{|c|}{$0,264^{* *}$} & \multicolumn{2}{|c|}{$0,121^{\star *}$} & \multicolumn{2}{|c|}{0,310 * } \\
\hline
\end{tabular}

*Teste Qui-quadrado de Pearson;**Teste Exato de Fisher

Legenda: PROLEC = Provas de Avaliação dos Processos de Leitura; TPF = Teste de Padrão de Frequência; TPD = Teste de Padrão de Duração; N = número de participantes; $\%$ = percentual

(estruturas gramaticais) $^{(21)}$. Desse modo, é possível afirmar que o baixo desempenho apresentado na leitura de palavras e pseudopalavras reflete a dificuldade na consciência fonológica e, consequentemente, na dificuldade para realizar a conversão grafema-fonema ${ }^{(21)}$. Por isso, faz-se necessária não somente a avaliação da capacidade de leitura geral, mas dos processos léxico, sintático e semântico, a fim de se identificar as dificuldades apresentadas na leitura e o nível em que ocorrem ${ }^{(22)}$.

Quanto à habilidade de ordenação temporal simples, assim como em estudo prévio ${ }^{(23)}$, a maior frequência de alterações ocorreu no TMSV em sequência. Uma possível explicação para tal achado é que, tanto no presente estudo, quanto no estudo citado $^{(23)}$, os participantes com dificuldades de aprendizagem foram os que mais apresentaram alterações no processamento auditivo. Cabe ainda ressaltar que a avaliação da ordenação temporal simples é utilizada como triagem do processamento auditivo, por ser rápida e de fácil aplicação.

Em relação à ordenação temporal complexa, o presente estudo indicou alteração em aproximadamente três quartos da amostra e, assim como em um estudo brasileiro ${ }^{(17)}$, os melhores desempenhos ocorreram nas tarefas envolvendo duração, quando comparadas às tarefas de frequência, tanto para as sequências de três como de quatro sons. Esse achado pode ser justificado pela própria fonética da língua portuguesa: há uma hipótese de que em razão de os fonemas no português possuírem duração (no que se refere à acústica) maior do que em outros idiomas, isso propicie o melhor desempenho na resolução de duração, quando comparada à resolução de frequência ${ }^{(16)}$. Além disso, um achado do presente estudo se diferenciou da literatura nacional ${ }^{(16,17)}$ : o maior número de acertos no TPD quatro sons, já que nos outros estudos ${ }^{(16,17)}$, o número de acertos foi maior no TPD três sons. Cabe ressaltar que os testes foram aplicados sempre iniciando pelo TPD 3 sons para, logo em sequência, aplicar o TPD 4 sons. Por esse motivo, levantou-se a hipótese de um possível efeito da aprendizagem do teste. Ou seja, o participante aprendeu a tarefa com o teste de 3 sons e, por isso, obteve melhor desempenho com o teste de 4 sons.

$\mathrm{Na}$ análise de associação do resultado do PROLEC com a idade, os dados encontrados neste estudo, embora não tenham apresentado significância estatística, confirmam estudo prévio ${ }^{(24)}$, ao demonstrarem piores resultados em participantes mais novos - neste caso, na faixa etária de 9 anos. Isso pode ser explicado porque se espera que, com o avanço da idade, o melhor uso da rota lexical, o aumento da fluência e da compreensão proporcione melhor desempenho durante a leitura ${ }^{(24)}$.

Quanto à análise de associação do resultado do PROLEC com a escolaridade, a maior frequência de alterações ocorreu em participantes do quarto, sexto e sétimo anos. Tal achado diverge do encontrado por outros autores ${ }^{(25)}$, uma vez que se espera que a partir do quarto ano, em decorrência do maior domínio da ortografia, a rota lexical seja mais utilizada e, consequentemente, a leitura mais rápida e precisa.

Acredita-se que na associação do PROLEC com a idade e a escolaridade, a ausência de significância estatística pode ser explicada pelo tamanho da amostra. Considerando que a amostra do presente estudo foi pequena, a estratificação por idade e escolaridade gerou grupos com números reduzidos de participantes, o que dificultou encontrar resultados mais robustos, com presença de significância estatística.

Em relação à análise de associação do PROLEC com a motivação escolar, o presente estudo indicou que a maior parte dos participantes com desempenho alterado nos processos de leitura apresentou motivação escolar média. Contudo, é pertinente ressaltar a subjetividade do teste que avalia a motivação escolar, o que pode ter contribuído para tais resultados. Apenas a associação da prova 7 (Estruturas gramaticais) com a motivação escolar geral apresentou significância estatística. Tal achado diverge do encontrado em outras pesquisas ${ }^{(6,26)}$, que demonstraram que estudantes motivados possuem melhor desempenho acadêmico e, também, maior motivação e melhores resultados nas tarefas que envolvem leitura. Cabe também evidenciar que a motivação 
pode sofrer influências de diversos fatores externos, dentre eles, as questões socioeconômicas e emocionais ${ }^{(26)}$.

No que se refere à associação do subteste de leitura do TDE com o PROLEC, o presente estudo identificou que a maioria dos participantes com alteração do desempenho nos processos de leitura também apresentou baixo desempenho escolar para o referido subteste do TDE. Embora apenas duas provas do PROLEC (prova 2: Igual-diferente em palavras e pseudopalavras e prova 7: Estruturas gramaticais) tenham apresentado significância estatística nessa associação, esse dado vai ao encontro dos achados de outro estudo, que afirma que a leitura é uma das bases para o aprendizado de diversas áreas do conhecimento ${ }^{(5)}$ e dificuldades para realizá-la podem levar a lacunas na aprendizagem de diferentes disciplinas escolares e, por conseguinte, ao baixo desempenho escolar ${ }^{(4)}$.

Em relação à associação entre o PROLEC e a habilidade auditiva de ordenação temporal simples, este estudo evidenciou que mais de dois quintos da amostra com adequação dos resultados nos testes de processamento auditivo apresentaram resultado alterado na leitura. A única exceção foi na associação entre a prova 5 (Leitura de pseudopalavras) e o teste de memória sequencial verbal, em que a maioria dos participantes com alteração na leitura também apresentou inadequação na ordenação temporal simples para sons verbais. Apesar de apenas a associação de uma prova do PROLEC com a ordenação temporal simples ter significância estatística, os achados concordam com a literatura $^{(9,27)}$ ao evidenciar que alterações nas habilidades auditivas são frequentemente relacionadas a dificuldades na leitura, escrita e aprendizagem. Uma possível explicação para a presença de significância estatística nessa associação é que, para a leitura de pseudopalavras, a memória sequencial verbal se faz ainda mais necessária para realizar a decodificação, já que não há como buscar no léxico a memória das pseudopalavras.

Por fim, no que se refere à associação entre PROLEC e ordenação temporal complexa, os resultados encontrados no presente estudo confirmam os achados de outros autores, que encontraram presença frequente de alterações das habilidades auditivas, em especial do processamento temporal, em participantes com alteração na leitura e aprendizagem ${ }^{(28,29)}$.

Além disso, cabe destacar a importante relação entre o processamento auditivo temporal e a leitura. As habilidades auditivas temporais desempenham um papel essencial, tanto para a fala, como para a compreensão da linguagem e, quando alteradas, podem refletir em dificuldades ortográficas e na codificação/decodificação, tanto de palavras, como de frases ${ }^{(9,30)}$.

Os achados do presente estudo constituem-se em um avanço ao discutir o desempenho de crianças e adolescentes em idade escolar em tarefas de processos de leitura (PROLEC) com habilidades auditivas de ordenação temporal simples e complexa. Deste modo, vale enfatizar que esta pesquisa abre caminhos para que a continuidade de estudos nessa área seja utilizada para a promoção de saúde no âmbito escolar, bem como para o aperfeiçoamento da intervenção na população que apresenta dificuldades nessas habilidades.

Por se tratar de um estudo com uma amostra reduzida, os resultados não puderam ser extrapolados para outro contexto. Além disso, a não equivalência da distribuição dos sujeitos nos grupos de faixa etária e escolaridade impossibilitou a realização de análises de associações mais robustas entre os dados.

Ademais, a presente pesquisa demonstrou a viabilidade do estudo e a efetividade dos instrumentos utilizados na coleta. Contudo, faz-se necessária a sua continuidade com amostras mais robustas, para uma investigação mais completa, tendo em vista o propósito de incorporar os resultados à prática fonoaudiológica e educacional.

Salienta-se a importância do estudo ao investigar a associação entre leitura, processamento auditivo temporal, queixas e motivação escolar, uma vez que a inter-relação desses aspectos é essencial para o desenvolvimento adequado das crianças e adolescentes. Espera-se, com este estudo, contribuir para que cada vez mais se incluam avaliações das habilidades auditivas em pacientes com dificuldade escolares, a fim de que as estratégias terapêuticas sejam mais assertivas e eficazes. Além disso, almeja-se, também, contribuir para a disseminação do conhecimento para os professores e educadores para que, uma vez conhecendo sobre a associação entre esses aspectos tão relevantes para a aprendizagem, possam auxiliar as crianças e adolescentes a obter desempenho escolar satisfatório.

\section{CONCLUSÃO}

O estudo evidenciou que há relação entre a variável resposta (processos de leitura) e as variáveis explicativas (habilidade auditiva de ordenação temporal, dados sociodemográficos e aspectos escolares). Contudo, não indicou significância estatística na maioria das associações.

\section{REFERÊNCIAS}

1. Uvo MFC, Germano GD, Capellini AS. Desempenho de escolares com transtorno do déficit de atenção com hiperatividade em habilidades metalinguísticas, leitura e compreensão leitora. Rev CEFAC. 2017;19(1):7-19. http://dx.doi.org/10.1590/1982-0216201719115815.

2. Bovo EBP, Lima RF, Silva FCP, Ciasca SM. Relações entre as funções executivas, fluência e compreensão leitora em escolares com dificuldades de aprendizagem. Rev Psicopedagogia. 2016;33(102):272-82.

3. Salles JF, Piccolo LR, Zamo RS, Toazza R. Normas de desempenho em tarefa de leitura de palavras/pseudopalavras isoladas (LPI) para crianças de $1^{\circ}$ ano $7^{\circ}$ ano. Estud Pesqui Psicol. 2013;13(2):397-419.

4. Oliveira KL, Boruchovitch E, Santos AAA. Leitura e desempenho escolar em português e matemática no ensino fundamental. Rev Paidéia. 2008;18(41):531-40. http://dx.doi.org/10.1590/S0103$863 \times 2008000300009$.

5. Vieiro P, Amboage I. Relación entre habilidades de lectura de palabras y comprensión lectora. Rev de Investigación en Logopedia. 2016;1(1):121. http://dx.doi.org/10.5209/rlog.58552.

6. Cartwright KB, Marshall TR, Wray E. A longitudinal study of the role of reading motivation in primary students' reading comprehension: implications for a less simples view of reading. Read Psychol. 2015;37(1):55-91. http://dx.doi.org/10.1080/02702711.2014.991481.

7. ASHA: American Speech-Language-Hearing Association. (Central) Auditory processing disorders [Internet]. Rockville: ASHA; 2005 [acesso em 15 mar 2020]. Technical report. Disponível em: http:// www.asha.org/policy/TR2005-00043.htm

8. Nishihata R, Vieira MR, Pereira LD, Chiari BM. Processamento temporal, localização e fechamento auditivo em portadores de perda auditiva unilateral. Rev Soc Bras Fonoaudiol. 2012;17(3):266-73. http://dx.doi.org/10.1590/S1516-80342012000300006. 
9. Rezende BA, Lemos SMA, Medeiros AM. Aspectos temporais auditivos de crianças com mau desempenho escolar e fatores associados. CoDAS. 2016;28(3):226-33. http://dx.doi.org/10.1590/2317-1782/20162015170. PMid:27462731.

10. Neves IF, Schochat E. Maturação do processamento auditivo em crianças com e sem dificuldades escolares. Pró-Fono Rev Atual Cient. 2005;17(3):311-20. http://dx.doi.org/10.1590/S0104-56872005000300005.

11. ABEP: Associação Brasileira de Empresas de Pesquisa. Critério de Classificação Econômica Brasil - 2018 [Internet]. 2018 [cited 2007 Jan 5]. Disponível em: http://www.abep.org

12. Lopes AC, Munhoz GS, Bozza A. Audiometria tonal liminar e de Altas Frequências. In: Boéchat EM, Menezes PD, Couto CM, Frizzo ACM, Scharlah RC, Anastasio ART, organizadores. Tratado de audiologia. 2. ed. São Paulo: Santos; 2015. p. 57-67.

13. Conselho Federal de Fonoaudiologia. Guia de Orientações na Avaliação Audiológica Básica [cited 2007 Jan 5]. 2021. Disponível em: http:// www.fonoaudiologia.org.br/cffa/wp-content/uploads/2013/07/Manualde-Audiologia.pdf

14. Pereira LD, Schochat E. Testes Auditivos Comportamentais para Avaliação do Processamento Auditivo Central. Barueri: Pró-Fono; 2011.

15. Gimenes VD. Identificação de padrões acústicos de duração e de frequência e habilidade grafo-fônica [tese]. São Paulo (SP): Universidade Federal de São Paulo; 2000.

16. Terto SSM, Lemos SMA. Aspectos temporais auditivos em adolescentes do $6^{\circ}$ ano do ensino fundamental. Rev CEFAC. 2013;15(2):271-86. http://dx.doi.org/10.1590/S1516-18462012005000040.

17. Nascimento LS, Lemos SMA. A influência do ruído ambiental no desempenho de escolares nos Testes de Padrão Tonal de Frequência e Padrão Tonal de Duração. Rev CEFAC. 2012;14(3):390-402. http:// dx.doi.org/10.1590/S1516-18462011005000093.

18. Stein LM. TDE: Teste de Desempenho Escolar: manual para a aplicação e interpretação. São Paulo: Casa do Psicólogo; 1994.

19. Cuetos F, Rodrigues B, Ruano E. PROLEC: Provas de Avaliação dos Processos de Leitura. 3. ed. São Paulo: Casa do Psicólogo; 2014.

20. Martinelli SC, Sisto FF. Escala para Avaliação da Motivação Escolar Infantojuvenil (EME-IJ). São Paulo: Casa do Psicólogo; 2011.
21. Oliveira AM, Cardoso MH, Capellini SA. Caracterização dos processos de leitura em escolares com dislexia e distúrbio de aprendizagem. Rev Soc Bras Fonoaudiol. 2012;17(2):201-7. http://dx.doi.org/10.1590/ S1516-80342012000200017.

22. Oliveira AM, Santos JLF, Vega FC, Capellini SA. Tradução e adaptação cultural da Bateria de Avaliação dos Processos de Leitura - PROLECSE-R. CoDAS. 2019;32(1):e20180204. http://dx.doi.org/10.1590/23171782/20192018204. PMid:31851209.

23. Pelitero TM, Manfredi AKDS, Schneck APC. Avaliação das habilidades auditivas em crianças com alterações de aprendizagem. Rev CEFAC. 2010;12(4):662-70. http://dx.doi.org/10.1590/S1516-18462010005000062.

24. Oliveira AM, Capellini SA. School-aged children's performance in the Brazilian adaptation of the reading processes assessment. Pro Fono. 2010 Out-Dez;22(4):555-60. http://dx.doi.org/10.1590/S010456872010000400033. PMid:21271116.

25. Oliveira AM, Germano GD, Capellini SA. Desempenho de escolares em provas de processo de identificação de letras e do processo léxico. Rev CEFAC. 2016;18(5):1121-32. http://dx.doi.org/10.1590/19820216201618523315 .

26. Paiva MLMF, Boruchovitch E. Orientações motivacionais, crenças educacionais e desempenho escolar de estudantes do ensino fundamental. Psicol Estud. 2010;15(2):381-9. http://dx.doi.org/10.1590/S141373722010000200017

27. Amaral MIR, Martins PMF, Colella-Santos MF. Temporal resolution: assessment procedures and parameters for school-aged children. Braz J Otorhinolaryngol. 2013;79(3):317-24. http://dx.doi.org/10.5935/18088694.20130057. PMid:23743747.

28. McDermott EE, Smart JL, Boiano JA, Bragg LE, Colon TN, Hanson EM, et al. Assessing auditory processing abilities in typically developing school-aged children. J Am Acad Audiol. 2016;27(2):72-84. http:// dx.doi.org/10.3766/jaaa.14050. PMid:26905528.

29. Santos TSL, Câmara CC, Moreira DR, Borges LL. Processamento Auditivo Central em crianças com dificuldades acadêmicas: revisão bibliográfica. Estudos. 2015;42(3):327-43.

30. Mourão AM, Esteves CC, Labanca L, Lemos SMA. Desempenho de crianças e adolescentes em tarefas envolvendo habilidade auditiva de ordenação temporal simples. Rev CEFAC. 2012;14(4):659-68. http:// dx.doi.org/10.1590/S1516-18462011005000141. 